\title{
The screaming balloon: predicting the sound produced by a hex nut inside a balloon
}

\author{
Henrique Ferreira, Matheus Pessoa*, Ricardo R. Gitti, André J. Ferreira-Martins, Gustavo de \\ Souza, Andrius Dominiquini, Lucas Coimbra, and Lucas Rios
}

Universidade Federal do ABC - 5001, Avenida dos Estados, Santo André, São Paulo, Brazil

Received: 2 November 2018 / Accepted: 11 August 2019

\begin{abstract}
In this work we studied the sound produced by the movement of a hex nut inside an air balloon. We demonstrated, by experiments with hex nuts and nuts without edges, the importance of the nut edges for the production of the screaming sound. Our experimental results show that the most intense frequency of the sound spectrum is directly proportional to the hex nut's speed of translation, while inversely proportional to its lateral length, thus allowing one to predict the most intense frequency generated by this system.
\end{abstract}

\section{Introduction}

Party balloons are present in people's day-to-day lives. Although they seem banal, they may be associated with very interesting physical phenomena, like its bursting $[1,2]$. Another interesting phenomenon is the screaming balloon: a sound produced by a hex nut rolling inside an air balloon.

The objective of our work is to describe the origin of that sound in this simple system: an air balloon (like a membrane of a spherical shell) with a hex nut inside it. The balloon is then rotated by a periodical mechanical movement source rotating the nut and therefore producing a specific screaming sound.

Many studies have also explored the vibration modes on spherical shells [3-5]. Horace Lamb was one of the first to mathematically develop these studies [3]. In [5], authors explore the problem of the excitation of a membrane using elasticity equations and the theory of elastic shells of Kirchhoff-Love. However, the most correlated work to ours has been presented in the International Physicists' Tournament in 2018 by Amaury Barral [6] with the same object of study and experimental challenges. Nevertheless, there is a great difference between our proposed method, based on the excitation of the motion of the nut inside the balloon using the movement of a hand, while Barral uses an electromechanical apparatus (which adds ego-noise in our opinion). In addition, we observe the spectrum of sound for complete translations of the nut, while Barral observes the whole spectrum. In Methods, we will discuss these assumed approximations and

\footnotetext{
* e-mail: matheus.pessoa@aluno.ufabc.edu.br
}

the experimental procedures used and measurements that are further investigated with a theoretical-experimental comparison.

Then, we present the results obtained and a phenomenological expression based on the relation between the velocity of the nut and the most intense frequency peak of sound. In the Dead End section we present some experimental difficulties during our exploration. In the conclusion we reinforce the results by predicting the sound feature considering the important system parameters.

\section{Method}

To collect the experimental data, we used balloons full of air, with a nut inside it. We used three different hex nuts $\mathrm{A}, \mathrm{B}$ and $\mathrm{C}$, and a small cylinder $\mathrm{S}$ to simulate a nut without edges (see Fig. 1 and Tab. 1). Each nut has been used in tests with different volumes of balloons. To each test a new balloon was used, giving a total of 18 balloons: 4 for the nut A, 7 for the nut B, 5 for the nut $\mathrm{C}$ and 2 for the cylinder $\mathrm{S}$ (nut without edge).

In Table 1, the average external radius of the hex nuts refers to the average of the distance between sides and the distance between edges. For the $\mathrm{S}$ nut without edges, the average external radius corresponds to the radius of this cylinder.

We model each balloon as a spherical shell (as in [6]). For this, we have measured the equatorial, polar and diagonal circumferences, so we could better determine an average radius of the ideal spherical balloon equivalent to each real balloon. We have determined the mass of four balloons using a precision scale, which gave us an average 
Table 1. Characteristics of the nuts.

\begin{tabular}{lllll}
\hline Nut & Size & Mass $(\mathrm{g})$ & Lateral length $(\mathrm{mm})$ & Average external radius $(\mathrm{mm})$ \\
\hline A & $3 / 8 ”$ & $6.6270 \pm 0.0001$ & $8.0 \pm 0.1$ & $7.5 \pm 0.2$ \\
B & $5 / 16 ”$ & $4.7135 \pm 0.0001$ & $7.8 \pm 0.1$ & $6.9 \pm 0.2$ \\
C & M6 & $2.2122 \pm 0.0001$ & $5.6 \pm 0.1$ & $5.2 \pm 0.1$ \\
S & - & $8.2690 \pm 0.0001$ & - & $5.4 \pm 0.1$ \\
\hline
\end{tabular}

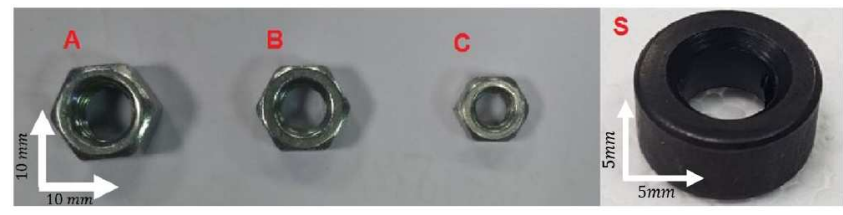

Fig. 1. Nuts used in our experiments.

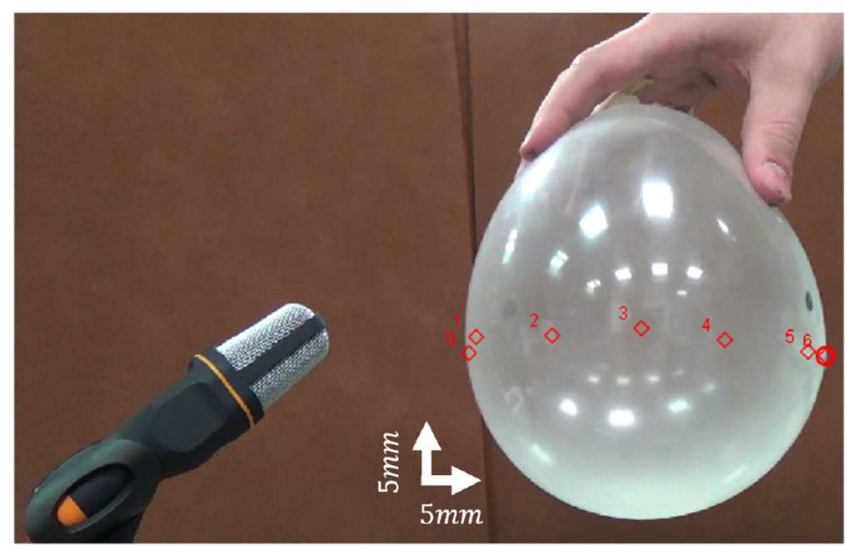

Fig. 2. Figure of the system analyzed via Tracker during our experiments. The distance between microphone and balloon was approximately the same for all experiments. The points show the trajectory of the hex nut while it passed in the frontal part of the balloon obtained using the Tracker software.

of $m_{b}=(1.3 \pm 0.1) \mathrm{g}$. This modelling was used to consider the trajectory followed by the nut as being proportional $2 \pi r_{b}$, where $r_{b}$ is the radius of the balloon.

To record the trajectory of the nut, the movement has been filmed by a camera and sound has been captured by a microphone. The experimental setup is presented in Figure 2.

Video and sound were synchronized using the Audacity software [7]. We used a transparent balloon so that one could record the nut trajectory. In order to observe the motion of the hex nut inside the balloon, we employed

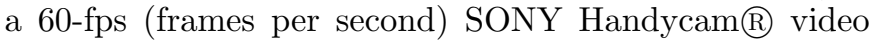
camera. The smallest timescale the camera can obtain is $0.017 \mathrm{~s}$ during video filming. To capture the sound, we employed a microphone of the brand TONOR, model SF666, connected to a computer.

Video data was analyzed with the Tracker software [8] to determine the velocity of translation of the nut. We can consider the sound processing with an error of approximately $11 \mathrm{~Hz}$ to determine the frequency, according to some specifications in [9].

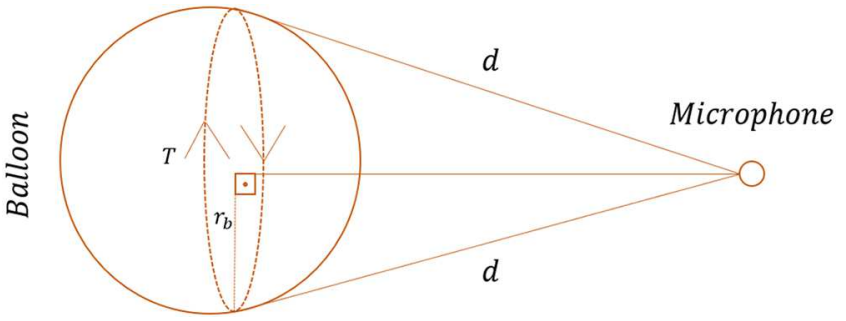

Fig. 3. Diagram considering the experimental condition that the distance $d$ between the plane of rotation of the nut and the microphone. $T$ is the trajectory of the nut and $r_{b}$ is the radius of the balloon.

The position of the microphone to capture the sound is of first importance. Every experiment may be modeled by the intersection of a cone with a sphere, where the sphere represents the balloon and the cone represents the geometric solid whose circular base is defined by the trajectory of center of mass of the nut within the balloon; and whose vertex is the point where the microphone is placed. A diagram illustrating this idea is presented in Figure 3.

In Figure 3, the distance between both extremities of the balloon and the microphone forms a cone, with generatrices $d$. If $d$ is equal for both extremities, the amplitude of the sound captured by the microphone is the same and thus we can infer about the translational velocity of the nut based in these measurements without further geometrical considerations to correct modulation or distance effects.

\section{Results}

\subsection{Importance of the edges}

The first question we address is the influence of the geometry of the nut. In order to answer this, we captured successively the sound produced by a balloon with a hexagonal nut and with the edgeless nut.

Regarding the hearing sensation, both sounds are completely different, with no characteristic screaming sound when the edgeless nut $\mathrm{S}$ is used (see Fig. 4a). It is shown in Figure $4 \mathrm{~b}$ that there is a significant difference between the intensity of the first peak when using a hexagonal nut (blue line) and when using the edgeless nut (red line).

\subsection{Frequency of the most intense peak}

We verified that during a period of 1 second the nut performs more than one turn. Therefore, for a full audio file of 

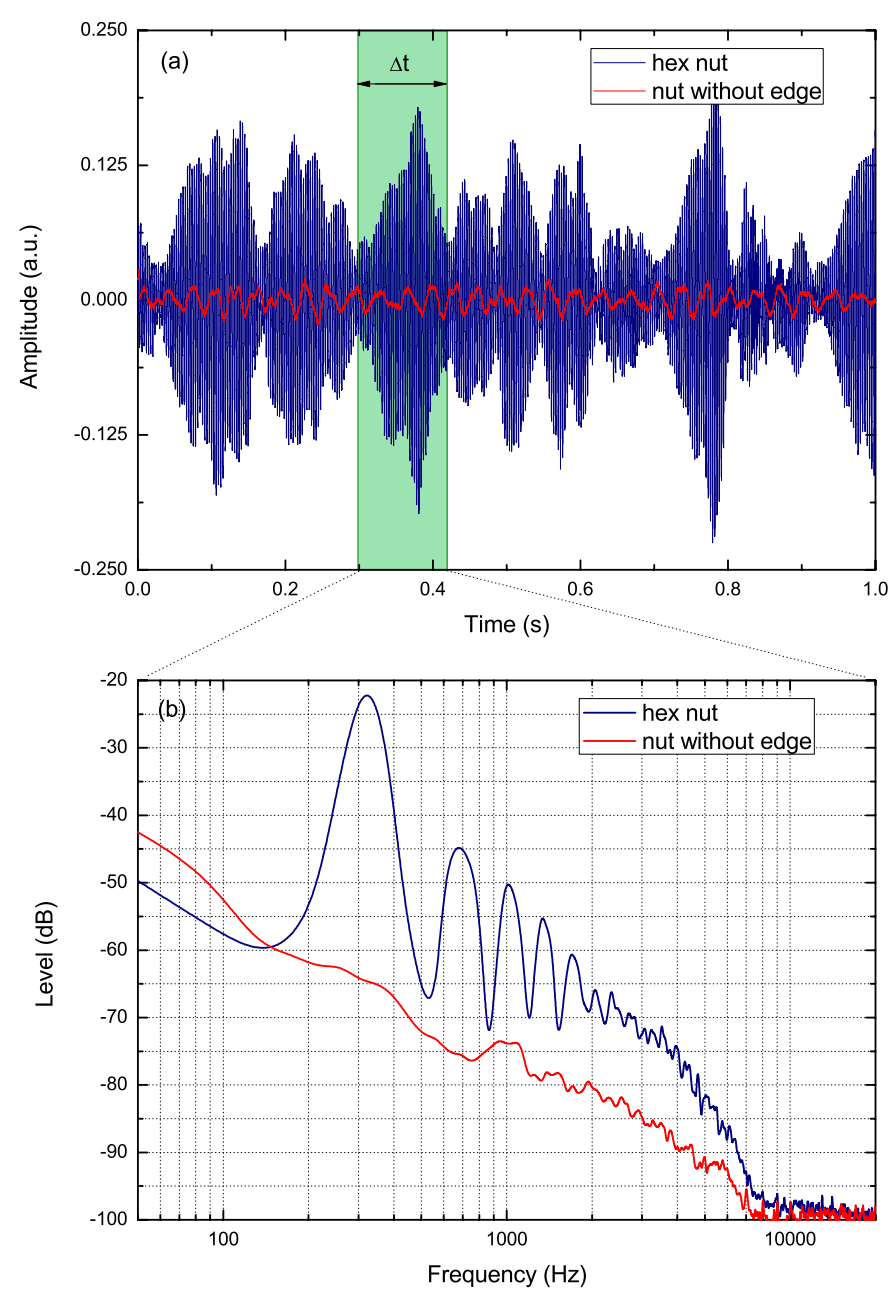

Fig. 4. (a) Signal in time domain of two audios. (b) Spectrum of a segment of these two audio of the same duration $(\Delta t)$. The blue line is for the nut $\mathrm{A}$ and a balloon of mean radius $(9.7 \pm 0.2) \mathrm{cm}$, with peak intensity of $(345 \pm 11) \mathrm{Hz}$, and red line is for the nut $\mathrm{S}$ and a balloon of mean radius $(9.1 \pm 0.2) \mathrm{cm}$.

10 seconds, there is a distribution of sounds caused by different translation velocities, whose variability is related to the non-constant trajectories of the nut, and the natural variability of the excitation of the system.

In the present study, we considered only hexagonal nuts and the time interval necessary for one complete translation. With the slow-motion analysis with the Tracker software, we determined the video frames corresponding to a complete translation with the synchronized sound capture, then selected the corresponding time interval in the sound file for the analysis of the sound frequency spectrum in the Audacity software. A typical spectrum is presented in Figure 4.

We selected four different audio tracks (audio segment representing a complete translation of the nut), and analyzed their respective region of total audio record, for each one of the 18 performed tests. Knowing the time interval $\Delta t$ necessary for the nut to complete one full translation within a balloon of mean radius $r_{b}$, we calculated the scalar translation velocity $v_{t}$ according to:

$$
v_{t}=\frac{2 \pi r_{b}}{\Delta t}
$$

For each studied interval, we selected the most intense frequency of the spectrum, as presented in Figure $4 \mathrm{a}$. The experimental results for every tested balloon and employed hexagonal nuts are presented in Figure 5a. The normalized velocity results by the nut lateral length of each experiment, are shown in Figure 5b. The bigger the lateral size of the hex nut, the smaller the travelled distance is. Thus we choose to plot in Figure $5 \mathrm{~b}$ the velocity renormalized by this parameter. In this figure, the dimensionless slope of the line is $(1.02 \pm 0.02)$, whilst the intercept is $(6 \pm 5) \mathrm{Hz}$.

\section{Discussion}

Figure 5 shows a strong experimental evidence that it is possible to know the most intense frequency produced by a nut with edges: it is caused by the local deformation induced by the edges of the nut as it rolls without sliding along the membrane. This can be inferred by looking at the non-normalized graph on the left, which presents a linear behavior for each hex nut lateral length: for the nut $\mathrm{A}$ and $\mathrm{B}$ this parameter almost does not change, changing only for the case of the nut $\mathrm{C}$ (see Tab. 1). This explains the distribution of the experimental points in Figure 5a: other parameters such as the mass of the nut and the average external radius are very different for the three nuts, which would not explain the almost equal distribution of the experimental points of the nut $\mathrm{A}$ and $\mathrm{B}$. It is observed that if the nut performed the sliding translation movement, the period between each perturbation on the balloon membrane should be different than that suggested by normalization by the nut side.

Now when considering the normalization of the results by the lateral length of the nuts, we get that the angular coefficient determines the proportionality relation between the sound most intense frequency $f_{\text {peak }}$ and the translation velocity $v_{t}$ divided by the nut side length $L$. That is,

$$
\frac{v_{t}}{L}=1.02 f_{\text {peak }}
$$

The linear coefficient obtained is related to the error of the experiment, since our precision in the frequency is $11 \mathrm{~Hz}$. The decimals presented in equation (2) can be neglected because the associated error of this parameter. In this way we can calculate the most intense frequency as

$$
f_{\text {peak }}=\frac{2 \pi r_{b}}{L \Delta t}
$$

This formula exhibits a simple phenomenological relation between the sound spectrum (most intense frequency) and the characteristics of the system (translation period, balloon radius and nut size). One way to understand this is by the ratio $\frac{2 \pi r_{b}}{L}$ which consists of the number of hex 

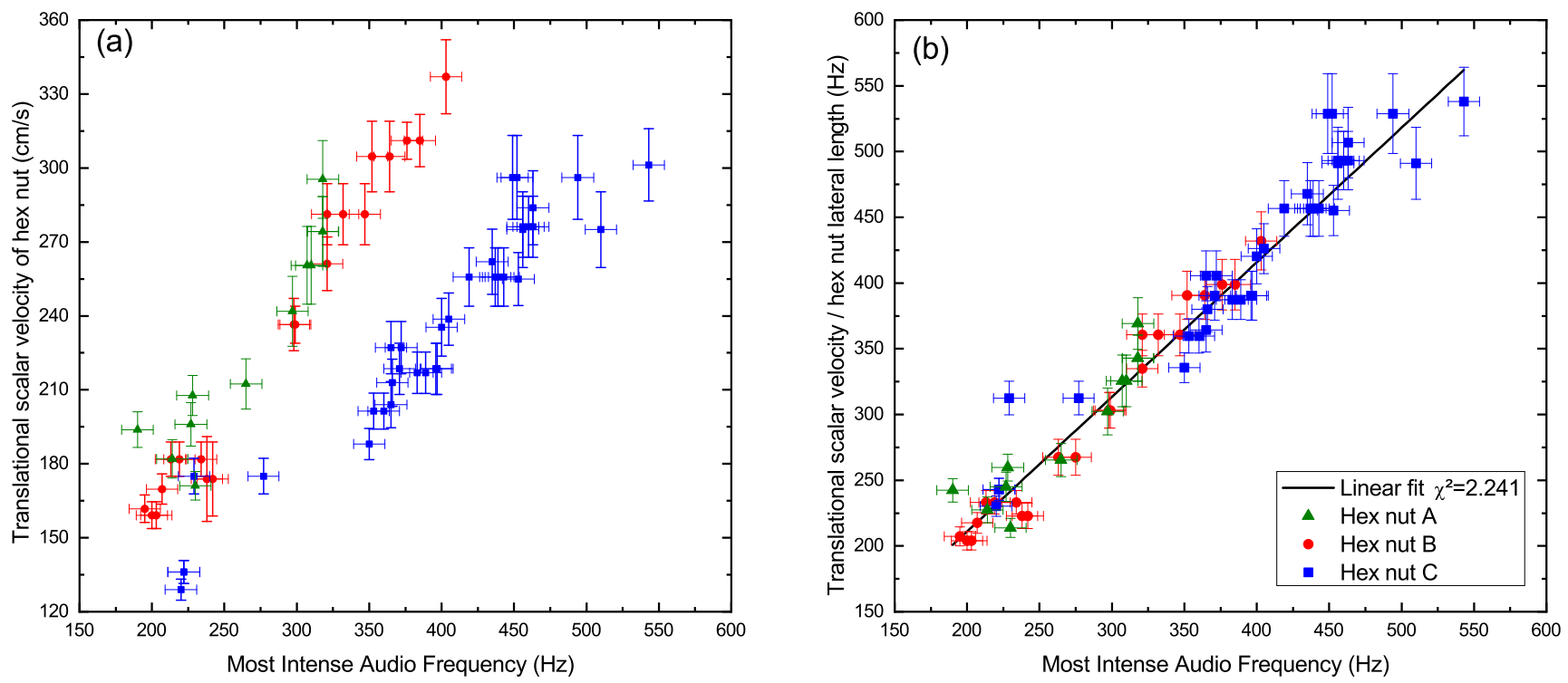

Fig. 5. Experimental results: (a) nut's translational velocity as a function of the most intense frequency in the audio signal and (b) normalized nut translational velocity as a function of the most intense frequency of the audio signal, whose slope is (1.02 \pm 0.02$)$ and the intercept is $(6 \pm 5) \mathrm{Hz} . \chi^{2}$ is the reduced chi-squared statistic that represents goodness of our fit.

nut rotations in a translation cycle. This is the number of times that the edge hits the balloon membrane in a $\Delta t$ period.

Considering that our experimental results were obtained with a hand-actuated balloon with different complex trajectories with a large range of translational velocities, we show how robust this model is in order to predict the sound produced.

\section{Dead end}

\subsection{Experimental difficulties}

We have tried a DC motor and an adapted drill as source of excitation. But we have not found a way to fix the balloon in this system in order to reproduce the phenomenon because the material of the balloon was too fragile to do this. Also, this type of devices contributes as another source of noise. Another difficulty was to determine the mechanical vibrations on balloon's membrane. A better camera, with higher fps, would be optimal for analyzing the relations between the deformation of the balloon in comparison to its equilibrium point and important parameters such as the nut velocity.

\subsection{Analogue model}

We tried modelling the problem using a simple damped harmonic oscillator but results showed us this approximation was mistaken due to the frequency modulation caused by the movement of the nut inside the balloon.

\section{Conclusion}

This work presents the experimental exploration on the problem of a screaming balloon, that happens whenever one puts a nut with edges inside a balloon and starts to rotate it. Our explanation for the source of the screaming (high frequency) is the rotation of the nut on its own axis bringing a repetitive movement in which the nut edges hit the balloon membrane, a hypothesis that was tested and confirmed experimentally considering the audio signals captured from a nut with and without edges. The strongest experimental result we present is a physical relation that allows one to predict the most intense frequency peak considering the radius of the balloon, the translation period and the lateral size of the nut inside it. As measuring a sound is easy, it could be a nice way to measure the nut velocity inside a more complex system that does not allow to use a tracking system, only knowing some characteristic lengths of the nut.

\section{References}

1. S. Moulinet, M. Adda-Bedia, Popping balloons: a case study of dynamical fragmentation, Phys. Rev. Lett. 115, 184301 (2015)

2. A. Stevenson, A.G. Thomas, On the bursting of a balloon, J. Phys. D: Appl. Phys. 12, 2101 (1979)

3. H. Lamb, On the vibrations of a spherical shell, London Math. Soc. 1, 50-56 (1882)

4. E.W. Ross Jr., Membrane frequencies for spherical shell vibrations, AIAA J. 6 (1968) 
5. Y.C. Chang and L. Demkowicz, Vibrations of a spherical shell comparison of 3-D elasticity and Kirchhoff shell theory results, Ticam Report (1994)

6. Slides presented by École Polytechnique, International Physicists' Tournament (2018). Available at: http://iptnet. info/faq/. Access at 09/08/2018
7. Audacity Website, Available at: https://www. audacityteam.org/. Access on: 07/18/2019

8. Tracker Website, Available at: https://physlets.org/ tracker/. Access on: 07/18/2019

9. Manual Audacity Spectrum, Available at: http://manual. audacityteam.org/man/plot_spectrum.html. Access on: $03 / 10 / 2018$

Cite this article as: Henrique Ferreira, Matheus Pessoa, Ricardo R. Gitti, André J. Ferreira-Martins, Gustavo de Souza, Andrius Dominiquini, Lucas Coimbra, Lucas Rios. The screaming balloon: predicting the sound produced by a hex nut inside a balloon, Emergent Scientist 3, 7 (2019) 